\title{
Relative Method of Measuring Coefficient of Luminous Intensity of Retro Reflective Devices
}

\author{
Eduard V. Kuvaldin \\ Metrology Department, S.I. Vavilov State Optical Institute, St. Petersburg, Russia \\ Email address: \\ ekuvaldin@yandex.ru \\ To cite this article: \\ Eduard V. Kuvaldin. Relative Method of Measuring Coefficient of Luminous Intensity of Retro Reflective Devices. American Journal of \\ Modern Physics. Vol. 7, No. 3, 2018, pp. 116-120. doi: 10.11648/j.ajmp.20180703.12
}

Received: May 15, 2018; Accepted: June 5, 2018; Published: July 7, 2018

\begin{abstract}
A relative method is proposed for measuring the coefficient of retro reflective light intensity CRLI through specific coefficient of retro reflective Luminance CRL and area of controlled product. In contrast to the adopted absolute measurement method, by measuring the ratio of light intensity of retro reflective sample to illuminance on it, it is proposed to measure reflectance of retro reflective sample in a small solid angle with calculation CRLI. The proposed method allows using the same testing unit and instruments for measuring CRLI, which are used to measure the CRL road signs and road markings. That permits to significantly reduce measurement error.
\end{abstract}

Keywords: Road Warning Triangles, Coefficient of Retro Reflective Light Intensity, Measurements, Reflectance, Testing Unit, Measurements Errors

\section{Introduction}

One of the main characteristics of retro reflecting materials is the specific coefficient of retro reflective luminance CRL, which determines the coefficient of retro reflectance of material under given illumination and observation conditions. For products made from retro reflective materials, such as bicycle retro reflecting element, road warning triangles, safety clothing labels, etc. the coefficient of retro reflective light intensity CRLI is measured. The measurement of CRL and CRLI in accordance with normative documents is made by an absolute method, i.e. measuring ratio luminance for CRL or ratio light intensity for CRLI to illuminance on retro reflective material

\section{Relative Method of Measurement}

The article proposes the relative measurement method of CRLI the same as for CRL [1]. The main advantages of relative method consider to example of measurement CRLI warning red colors triangles, ensuring safety on the road. According to UNECE regulation №27 with the 03 series of amendments (03.03.85 CRLI is the ratio of light intensity of warning triangle to illuminance on it when the angle of deviation of observer or deviation from optical axis of light source is equal to 20 minutes. In this case angular field of view of measuring device should not exceed 10 minutes. The deflection angle is determined by observation of a driver of vehicle. Angular field of view depends on focal length of lens luminance meter and size of field diaphragm in it. The maximum angle in case of observation of road markings is set to 20 minutes and is determined by width of layout and distance of observation which is equal $150 \mathrm{~mm}$ and $30 \mathrm{~m}$. In fact, a reflected from a triangle light flux at one point on reflectance indicatrix of reflective surface element of triangle is measured. This is a small indicatrix of retro reflective element, beyond which the element loses its retro reflective properties. The property of retro reflection one used materials and structures such as reflectors, is saved in a fairly wide viewing angle up to $40-50$ degrees pivot the reflective element relative to the optical axis of light source. The dependence of CRL from rotation angle of retro reflective device presented an indicatrix of efficiency, that is, conditionally, a large indicatrix. Values of angles on this indicatrix for measurement CRLI are also given in standard. The total angle under which the observer sees the whole triangle should not exceed 80 minutes. This imposes a restriction on the distance from which measurement CRLI must be made. So if the side of triangle is $360 \mathrm{~mm}$ this distance should be at least $20 \mathrm{~m}$, which causes certain 
difficulties in the construction of testing unit. This is not only dimensions of the unit, but also difficulty of obtaining a narrow illuminating beam from a type $A$ source [2] for receiving sufficient illumination at test sample. Increase the luminance of light source is not possible, as it limited color temperature of source type $A$. This suggests a natural remedy to overcome these difficulties. It is better to measure a flux reflected from a small reflective surface of triangle, then it is multiplied by ratio of luminous triangle area to the area of measured element surface. Then you can decrease specified distance. In this case is measured flux per unit surface in the angle of observation, i.e. luminance of a luminous surface. The ratio of retro reflective luminance of device to illuminance on it is CRL used in the control of road signs and road markings [3, 4]. So it is can used techniques and accepted methods of measurement of CRL.

In [1] proposed a relative method of measuring CRL, which is reduced to measure ratio of luminance's of measured surface and light source. In the proposed method of measurement, as in [1] when small angles and areas of receiver's elements are equal, so ratio of luminance's is equal to reflectance $R$ of reflective element of device. For correct measuring this ratio it is necessary to measure flux on the surface of element with area $A$ and flux radiated by this element in the same solid angle with the same area $A$. If in this case to choose distance from radiation source to surface of test triangle is such to withstand the required angle of deflection, such method can be measured CRL, using only one illuminance meter. The device measures illuminance on the sample, then measure illuminance near the illuminator, which is translated into luminance by dividing on value of solid angle of observation or the divergence of beams, defined by area of detector and distance from measured sample. In these measurements the calibration of illuminance meter in absolute units is not required. It is enough to have a wide range of measurement limits, which must be at least three orders of magnitude. Thus measured value of CRL converted to CRLI by multiplying by the ratio of area of triangle and area of receiving site of detector or diaphragm in front of it.

\section{Testing Unit}

Testing unit by the relative method of measurement consists of the illuminator with a glow lamp working in the mode of $A$ type source and the detector with correcting filter (Figure. 1). The spectral curve of detector is corresponded to the curve of international photometric observer $V \lambda$ [2]. It is desirable to take diameter of tube of illuminator as little as possible. It will allow reducing testing unit length. The detector is situated in a black tube closely to illuminator on 10 -. $20 \mathrm{~mm}$ farther from an output end of illuminator for scattering light elimination.

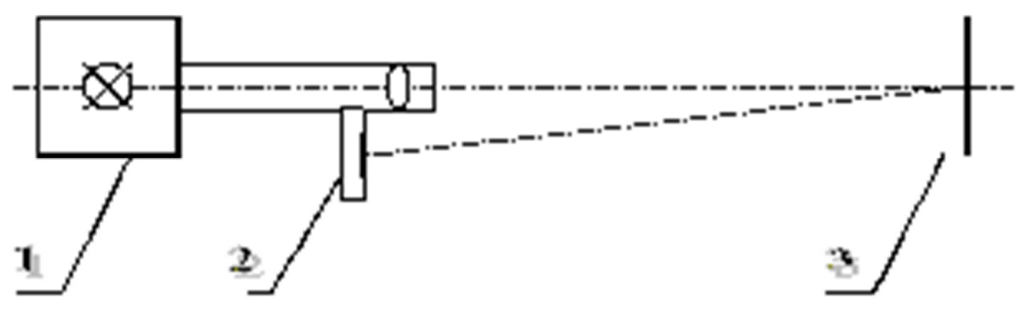

Figure 1. Scheme of testing unit: 1 - illuminator, 2 -detector, 3 - measured sample.

The test sample is placed on the turntable at a distance from illuminator $L$, determined by formula $L=d / \operatorname{tg} \omega$, where $d$ is distance between centers of illuminator and detector, $\omega$ is divergence angle equal in accordance with standard of 20 minutes. The detector with recording device must be able to switch the responsibility by at least 3 orders of measured value. This is usually achieved by switching detector which usually is a silicon photodiode, load resistors in feedback circuit of operational amplifier [5]. Applying a load resistor up to $100-300 \mathrm{M} \Omega$ and using photodiode without a bias voltage, it is possible to get illuminance meter lower limit to $110^{-5}$ lux.

If switching responsibility of illuminance meter by load resistors are not enough, it is possible to insert a neutral filter with a transmittance $\tau$ before detector. The measurements are carried out in two stages:

A diaphragm with an area equal to the area of detector receiving area is placed on measured warning triangle of safety on the road. The rest of the sample is covered with black material. The count $n 1$ take off when illuminance meter situated near illuminator. At the second stage, the illuninance meter is installed in location of diaphragm on the sample. If the receiving area of detector is larger than the area of diaphragm, the detector is placed behind diaphragm. The illuminfnce meter measurement limit is set so as to obtain an illumination reading from the illuminator $n 2$ with a minimum error. The sampling ratio $R=n 1 / n 2$ is the reflectance of measured sample in direction of illumination in a small solid angle $\omega$ determined by the distance from measured sample to detector at the location near illuminator and by the area of diaphragm before detector $A_{\mathrm{d}}$. The value of this coefficient divided on the value of solid angle $\omega$, is specific coefficient of retro reflection luminance or CRL, the same as in the case of measurement road signs and road markings, since it is measured at the same angle deviation of 20 minutes. Thus, the required coefficient of retroreflective luminous intensity CRLI can be calculated by formula CRLI $=R A / A_{\mathrm{d}} \omega=(n 1 /$ n2) $A / A_{\mathrm{d}} \omega$. Here $A$ is area of triangle. The value $1 / \omega$ calculated in advance and for deviation angle of 20 minutes it is equal to 37680 . When measured on standard-based devices by two instruments, this factor depends on design of luminance meter and it is automatically taken into account 
when calibrating it.

If a small signal is obtained during measurements, then to increase reflected signal, it is possible to reduce the distance, saving only deflection angle. Even more signal can be obtained by increasing the area of luminous part. In this case, the ratio of luminous area of triangle and area of detector or diaphragm before it is included in calculation. Finally, calculation formula looks like this

$$
\mathrm{CRLI}=A_{\mathrm{d}} / A_{\mathrm{i}}(1 / \omega)(n 1 / n 2) A .
$$

Where $A_{\mathrm{d}}$ is receiving area of detector, $A_{\mathrm{i}}$ is area of luminous part of triangle, $A$ is area of triangle, and $\omega$ is detector divergence angle. The factor $A_{\mathrm{d}} / A_{\mathrm{i}}(1 / \omega)$ should be calculated in advance for this measurements. Then it will be necessary to measure only reflectance and area of triangle i.e. standard measurements in photometry is used. The remaining requirements for measurement correspond to standard.

If the area of luminous part of triangle is increased, then due to violation of divergence angle, measurement error increases. However, the random error decreases, since detector's readings become more stable. Therefore, during measurements, it is necessary to select the optimum size of luminous part of triangle so as to obtain a minimum error in the measurements. The reflection coefficient obtained in the measurement characterizes properties of retro reflective material only at a deviation angle of 20 minutes, which is used in road conditions. In other applications, for example, when illuminated with scattering light on clothing, it is desirable to know the reflectance $R_{0}$ at deviation angle of zero. The reflectance $R_{0}$ is equal to the coefficient, called reflectance factor or directional reflectance. This coefficient in International Lighting Technical Dictionary [6] is defined as the ratio of light flux reflected in directions bounded by a given cone to the flux reflected in the same directions by perfect reflecting diffusor under the same lighting conditions. This coefficient differs from luminance factor by factor $\pi[1]$ for small angles, for which it is possible not to take into account the indicatrix of reflectance. Therefore, when developing a new retro reflective material or evaluating its properties during aging and external influences, it suffices to measure the coefficient $R_{0}$ with illumination and observation angles tending to zero and lead both indicatrices of reflection. In technical regulations for measuring methods, it is sufficient to indicate only maximum values of illumination and observation angles defining measurement errors. In the case of a visual assessment of perception of the retro reflective material operation under certain lighting and observation conditions, for example on an automobile road, the coefficient $\mathrm{R}_{0} / \omega$ is multiplied by the correction factor for specific values of deflection and divergence angles. As a result, CRL is determined by technical regulations for road equipment. The correction factor can be measured once in development of retro reflective material and included in its passport. Then, during further measurements coefficient $R_{0}$ or $R$ is measured and, if necessary, the values of CRL and CRLI, which are not universal characteristics of material are calculated. The universal characteristics in this case are the spectral reflectance $R_{0}$ for small angles and its indicatrices. For visual perception, the small and large indicatrices of luminance factor, which is proportional to CRL under the required measurement conditions, will be universal.

\section{Measurement Errors}

The main sources of error in measurement methods are:

- The RMS of the average arithmetic value of change in signal $S$ according to test results does not exceed 0.02 .

- the error of calibration of luminance meter and illuminance meter $\Theta_{1}$ and $\Theta_{1}$ according to the standard scheme Russian GOST 8.203-2007 and calibration experience [7-9] is 0.1 .

The error of $\Theta_{\mathrm{s}}$ from the inaccurate matching of spectral curve of corrective light filter to the spectral function $V_{\lambda}$ [2] is calculated below.

The error in setting the angles in the measuring unit $\Theta_{u}$ is no bigger than 0.005

Let's present the calculation of spectral error for the illuminator with a source of type $A$. The corrective filter adjusts spectral curve of detector to the curve $V_{\lambda}$ with some error. Correction error is usually evaluated against a type $A$ source integrally over whole spectrum. The error of correction for good detectors in this way is estimated to be 2$5 \%$. The difference in the spectral curve of corrected detector from the curve $V_{\lambda}$ is usually much larger, especially in red and blue regions of spectrum and can reach several times. Let's calculate what error can be in the case of measurement of red sample. Spectral transmission curve of glass of such a triangle roughly corresponds to the spectral transmission curve of the KS13 glass [10]. The spectral curves of three samples of actually manufactured detectors (Table 1 and Figure 2) show that the spectral curves of manufactured detectors are similar to each other and to curve $V_{\lambda}$

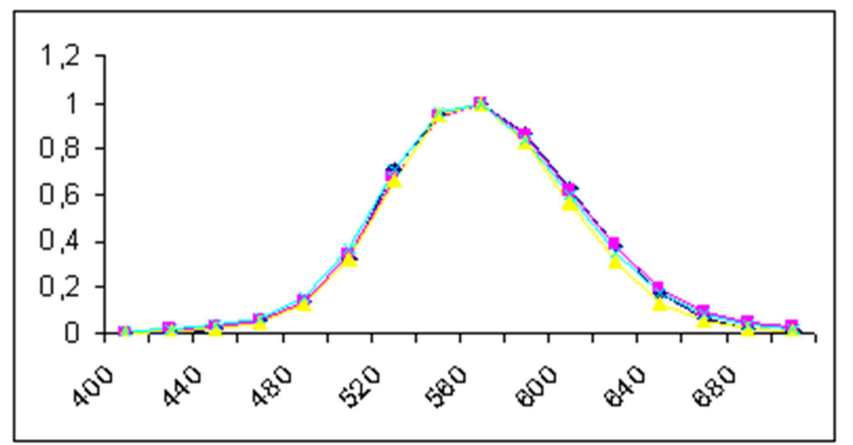

Figure 2. Dependence of detector responsibility from the wavelength of three detectors and curve $V_{\lambda}$.

Table 1. Spectral response of detectors and curve $V_{\lambda}$.

\begin{tabular}{lllll}
\hline $\boldsymbol{\lambda}$ & $\boldsymbol{V} \boldsymbol{\lambda}$ & detector $\mathbf{1}$ & detector $\mathbf{2}$ & detector $\mathbf{3}$ \\
\hline 400 & 0.0004 & 0.007 & 0.004 & 0.01 \\
420 & 0.004 & 0.017 & 0.01 & 0.023 \\
440 & 0.023 & 0.029 & 0.022 & 0.036 \\
460 & 0.06 & 0.057 & 0.049 & 0.066 \\
480 & 0.139 & 0.137 & 0.125 & 0.156 \\
500 & 0.323 & 0.344 & 0.323 & 0.371 \\
520 & 0.71 & 0.678 & 0.667 & 0.713 \\
\hline
\end{tabular}




\begin{tabular}{lllll}
\hline $\boldsymbol{\lambda}$ & $\boldsymbol{V} \boldsymbol{\lambda}$ & detector $\mathbf{1}$ & detector $\mathbf{2}$ & detector 3 \\
\hline 540 & 0.954 & 0.945 & 0.948 & 0.964 \\
560 & 0.995 & 1 & 1 & 1 \\
580 & 0.87 & 0.863 & 0.833 & 0.842 \\
600 & 0.631 & 0.617 & 0.566 & 0.599 \\
620 & 0.381 & 0.379 & 0.311 & 0.351 \\
640 & 0.175 & 0.192 & 0.125 & 0.174 \\
660 & 0.061 & 0.096 & 0.058 & 0.081 \\
680 & 0.017 & 0.046 & 0.02 & 0.034 \\
700 & 0.008 & 0.027 & 0.009 & 0.015 \\
\hline
\end{tabular}

To estimate the error in the correction, the spectral curves of detector and curve $\mathrm{V} \lambda$ were multiplied by the spectral distribution curve of radiation source of type $A$ and then summed.

After this, the ratio of sums received for detectors to sum for curve $V_{\lambda}$ is calculated.

Similarly, calculations were made of spectral component of measurement error for radiation of red color that passed through the KS13 glass filter [10] and white color (Table 2). Areas under spectral curves of detectors are proportional to the signals from them. Ratios of these areas to the area under detector with a curve $V_{\lambda}$ characterize the additional spectral component of measurement error. For a type $A$ source, this is spectral component of measurement error of light measuring devices. For white and red samples it is an additional spectral error relative to an ideal detector for light measurements. CRL and CRLI measurements are relative measurements. So

$$
\Theta= \pm \mathrm{k}\left(\Theta_{\mathrm{j}}^{2}+\Theta_{1}^{2}+\Theta_{\mathrm{s} 1}^{2}+\Theta_{\mathrm{s} 2}{ }^{2}+\Theta_{\mathrm{u}}{ }^{2}\right)^{1 / 2}=1.1
$$

Where $\Theta_{\mathrm{s} 1}$ and $\Theta_{\mathrm{s} 2}$ are errors of spectral components of luminance meter and illuminance meter

The basic measurement error of the CRLI, including the non-excluded systematic component of error $\Theta$ and the random component of measurement $S$ is:

$$
\Delta=2\left[\Theta_{\mathrm{c}}{ }^{2} / 3+S^{2}\right]^{1 / 2}=2\left[\left(0.15^{2}\right) / 3+0.02^{2}\right]^{1 / 2}=0.153
$$

In the relative method of measurement under consideration, there is no spectral component of overall measurement error and calibration error of illuminance meter and luminance meter. Then the basic error in measuring CRLI will have only a random component of reading $\mathrm{n} 1$ and $n 2$, as well as error in measuring divergence angle, which has two components: the error in measuring a distance between illuminator and sample being measured and measurement error of diaphragm area before detector.

RMS random component of sampling for measurement reflectance according to the verification scheme Russian GOST 8.557 - 91 for measuring instruments does not exceed $2 \%$. The errors in linear measurements are even smaller. The total error of measurement by the relative method will be no more than $2.5-3 \%$.

\section{Method Modernization}

To reduce the measurement error and simplify design of testing unit with a single detector, it is possible by using a laser as an illuminator. Reflectance of white samples in if luminance and illuminance measures through the same correcting light filter there is no spectral error.

Table 2. Ratio of area under spectral curves of detectors and area under curve $V_{\lambda}$ in spectral intervals of $A$ type source, white samples and red samples.

\begin{tabular}{llll}
\hline Detectors & A type source & White sample & Red sample \\
\hline Detector 1 & 1.026 & 1.0154 & 1.26 \\
Detector 2 & 0.946 & 0.947 & 0.82 \\
Detector 3 & 1.048 & 1.0156 & 1.088 \\
\hline
\end{tabular}

When different filters are used an additional spectral error defined by ratio of meanings for two detectors in Table 2.

For example, even at best, if the spectral curve of one of detectors in the standard-based testing unit corresponds to detector 1 and the other to detector 3 , the spectral component of error in measuring red retro reflective samples is $1.26 / 1.088=$ 1.16 , which corresponds to $16 \%$. For other combinations of detectors additional errors are $32 \%$ and $53 \%$. This phenomenon was found 40 years ago with serial production of luminance meters. A separate calibration for red samples was made in this case. In highly sensitive luminance meters photomultipliers are usually used, for which the error of correction is even greater. The same result was received with spectral response curve some red color LED. For white samples, the situation is much better. In this case, the total boundary of the non-excluded systematic error of measurement:

$$
\left(0.1^{2}+0.1^{2}+0.048^{2}+0.026^{2}+0.005^{2}\right)=0.151,
$$

visible region of spectrum is the same for all wavelengths, so a laser of any color is suitable. For red samples only a red laser is suitable, and red laser is suitable for all colors except blue and green. For these colors one can use an illuminator with blue LED and forming optical system [11, 12]. Reflective panels of pure blue or green color are practically not found. Usually they are multicolored and, as a rule, with sections of white color. Control of such panels can be carried out with a red laser in other colors. The blue light is only needed in stationary testing units, on which reference samples are calibrated.

The laser illuminator allows to exclude the spectral component of measurement error and to reduce the distance to measured sample. Due to the high brightness of laser, the responsibility of detector can be reduced and there is no need to use a corrective filter in it. This further reduces requirements to detector responsibility and simplifies its design. For simplified control in a field conditions, it is advisable to make a simplified version of testing unit, which is a portable device consisting of a laser illuminator and a detector near it. Next to the measured sample of traffic sign, warning trapper, security indicator, advertisement, etc. set a reference sample and from a distance of several meters consistently illuminate the reference and the measured sample. Knowing CRL of the reference sample through the ratio of obtained readings is calculated the CRL of sample being measured and, if necessary, also calculates the CRLI through the area of measuring sample. 


\section{Conclusions}

Retro reflection possibility of retro reflective materials must be measured through measuring reflectance in a small solid angle and area of sample with calculation CRL and CRLI in observation in a road conditions. Described relative method of measuring CRL is permits to decrease measuring errors. As can be seen from errors calculations, the measurement error by the standard-based technique for white samples is about $15 \%$. Red samples can be measured with a large additional error by this method. The measurement result for them can differ $50 \%$ and more from the true one. In the relative method under consideration, the measurement error does not exceed $2-3 \%$ for white and color samples. A corrective filter is not required, since the measurement light intensity and illumination is made by the same detector.

\section{References}

[1] Kuvaldin E. V. Relative method of measuring the photometric characteristics of road signs and marking / / Measuring technique. 2010. № 4. pp. 64-68.

[2] Russian GOST 7721 - 89 Light sources for measuring color.

[3] Kuvaldin E. V. "Equipment for measuring the coefficient of horizontal road marking" // Roads of Russia in the 21st century, 2005, 2, pp. 73-75.

[4] Kuvaldin E. V. Methods of measuring the coefficient of retro reflectance of road markings and road signs // International conference "Applied optics" St. Petersburg November 2006, Volume 1, page 248-251.

[5] Kuvaldin E. V. Meters of energy and power of optical radiation. // International Conference "Applied Optics" St. Petersburg, November 2006, Volume 1, pp. 217-221.

[6] International Lighting Dictionary // Publisher Russian language, M., 1978, $278 \mathrm{p}$.

[7] E. V. Kuvaldin: Method of calibrating pulse photometers of energy and power with conversion in time // Journal of Optical Technology, Volume 73. 18, 2006 pp. 42-47.

[8] E. Kuvaldin: Counting Method for Measuring and Linearity Checking Photometry Devices //Measurement science review http://www.measurement.sk/2010/S1/p.html and in: VersitaCentral European Science Publisher: http://versita.metapress.com/content/121037/, Volume 10, Number $2 / 2010$

[9] Kuvaldin E. V. Optical Energy meters and sources of radiations calibrations in a wide measuring range/ / Measuring technique. 2018. in press.

[10] Catalog of colored glass // Mechanical engineering, M, 1967, $63 \mathrm{p}$.

[11] E. V. Kuvaldin: Remote and contact devices of plant condition diagnostics / / Journal of optical technology. 2013. No 11. pp. 71-80.

[12] Kuvaldin E. V., Shulga A. A. Pulsed light-emitting diode emitter // Journal of optical technology, 2017. No. 9, pp. 8589. 